\title{
Path Integral Quantization of Doubly Supersymmetric Model
}

\author{
Hanaa Abdulkareem Elegla, Nasser Ismail Farahat \\ Physics Department, Islamic University of Gaza, Gaza, Palestine \\ Email: helegla@iugaza.edu.ps, nfarahat@iugaza.edu.ps
}

How to cite this paper: Elegla, H.A. and Farahat, N.I. (2022) Path Integral Quantization of Doubly Supersymmetric Model. Journal of Applied Mathematics and Physics, 10, 245-253.

https://doi.org/10.4236/jamp.2022.102018

Received: November 28, 2021

Accepted: February 6, 2022

Published: February 9, 2022

Copyright $\odot 2022$ by author(s) and Scientific Research Publishing Inc. This work is licensed under the Creative Commons Attribution International License (CC BY 4.0).

http://creativecommons.org/licenses/by/4.0/

\begin{abstract}
The Hamilton-Jacobi formalism is used to discuss the path integral quantization of the double supersymmetric models with the spinning superparticle in the component and superfield form. The equations of motion are obtained as total differential equations in many variables. The equations of motion are integrable, and the path integral is obtained as an integration over the canonical phase space coordinates.
\end{abstract}

\section{Keywords}

Hamilton-Jacobi Formalism, Singular Lagrangian, Superparticle, Superfield, Supersymmetry

\section{Introduction}

Supersymmetric particles "superparticles" were stimulated by dynamical developing research in the supersymmetry in the present decade with such new models presented by: Brink-Schwarz [1] and Siegel [2]. This aspect of the superparticle models makes that they are instructive toy models used to understand the superstrings and the variety of their covariant quantization procedures [3] [4]. It is known that the puzzle of covariant quantization of the model can be addressed in two respects: the problem of quantizing the infinitely reducible first-class constraints, and the problem of quantizing the infinitely reducible second-class constraints. Since the first-class constraints trend to reduce (directly or indirectly) the phase space eliminating the constraints, while, the second trend, quite opposite to the first one, implies extending the initial phase space by auxiliary variables to convert the original second-class constraints into effective first-class ones in the extended manifold [1] [5] [6]. The Hamiltonian formulation of constrained systems was initiated by Dirac [7] [8]. The main feature of this method 
is to consider primary constraints initially, then all constraints are obtained by using the consistency conditions. Hence the equations of motion of a singular Lagrangian system are obtained by the consistency conditions. An alternative Hamilton-Jacobi formalism for constrained systems, based on Carathéodory's equivalent Lagrangians methods [9] is developed by GÜler [10] [11]. In Hamilton-Jacobi formalism, we have no difference between first and second class constraints and we do not need gauge-fixing term because the gauge variables are separated in the processes of constructing an integrable system of total differential equations. The path integral quantization serves as a basis to develop perturbation theory and to find out the Feynman rules. The path integral quantization of singular theories with first-class constrains in canonical gauge was given by Faddeev and Popov [12]. The generalization of the method to theories with second-class constraints is given by Senjanovic [13]. Moreover, Fradkin and Vilkovisky [14] [15] considered quantization to bosonic theories with first-class constraints and it is extension to include fermions in the canonical gauge. When the constrained dynamical system possesses some second-class constraints there exists another method given by Batalain and Fradkin the BFV-BRST operator quantization method, which implies extending the initial phase space by auxiliary variables to convert the original second-class constraints into effective first-class ones in the extended manifold. Recently, a new scheme of path integral quantization [16]-[24], depends on the Hamilton-Jacobi treatment of constrained systems. According to Hamilton-Jacobi formalism, the equations of motion are obtained as total differential equations in many variables which require investigating the integrability conditions. The canonical path integral quantization is obtained directly as integration over the canonical phase-space coordinates without any need to enlarge the initial phase-space by introducing extra-unphysical variables. The advantage of the Hamilton-Jacobi formalism is that we have no difference between first and second class constraints and we do not need gauge-fixing term to reduce or enlarge the physical phase-space. A better understanding of these features arises by applying the Hamilton-Jacobi formalism for supersymmetric constraint systems [25]-[30], which are subject to mixed fermionic first and second-class constraints in an arbitrary space-time dimension.

The work is organized as follows: In Section 2, Hamilton-Jacobi Formulation is presented. The motion doubly supersymmetric model [31] [32] [33] is analyzed by using Hamilton-Jacobi Formulation and the path integral is obtained as an integration over the canonical phase space coordinate in Section 3. In section 4 , the conclusion is given.

\section{Hamilton-Jacobi Formalism of Constrained Systems}

The system that is described by the Lagrangian $L\left(q_{i}, \dot{q}_{i}, t\right), i=1, \cdots, n$, is constrained system if the Hessian matrix

$$
A_{i j}=\frac{\partial^{2} L}{\partial \dot{q}_{i} \partial \dot{q}_{j}} \quad i, j=1, \cdots, n,
$$


has a rank $(n-r), r<n$. In this case, we have $r$ momenta are dependent on each other. The generalized momenta $p_{i}$ corresponding to the generalized coordinates $q_{i}$ are defined as,

$$
\begin{gathered}
p_{a}=\frac{\partial L}{\partial \dot{q}_{a}} \quad a=1, \cdots, n-r, \\
p_{\mu}=\frac{\partial L}{\partial \dot{q}_{\mu}} \quad \mu=n-r+1, \cdots, n
\end{gathered}
$$

Since the rank of the Hessian matrix is $(n-r)$, one may solve (2) for $\dot{q}_{a}$ as

$$
\dot{q}_{a}=\omega_{a}\left(q_{i}, \dot{q}_{\mu}, p_{a}\right) .
$$

Substituting (4) into (3), we obtain relations in $q_{i}, p_{a}, \dot{q}_{v}$ and $t$ in the form

$$
p_{\mu}=\left.\frac{\partial L}{\partial \dot{q}_{\mu}}\right|_{\dot{q}_{a}=\omega_{a}}=-H_{\mu}\left(q_{i}, \dot{q}_{v}, \dot{q}_{a}=\omega_{a}, p_{a}, t\right) .
$$

The canonical Hamiltonian $H_{0}$ is defined as

$$
H_{0}=-L\left(q_{i}, \dot{q}_{v}, \dot{q}_{a}=\omega_{a}, t\right)+p_{a} \omega_{a}+\left.\dot{q}_{\mu} p_{\mu}\right|_{p_{v}=-H_{v}} .
$$

The set of Hamilton-Jacobi partial differential equations (HJPDE) is expressed as

$$
H_{\alpha}^{\prime}\left(q_{\beta} ; q_{a} ; p_{a}=\frac{\partial S}{\partial q_{a}} ; p_{\alpha}=\frac{\partial S}{\partial q_{\alpha}}\right)=0, \alpha, \beta=0, n-r+1, \cdots, n,
$$

where

$$
\begin{aligned}
& H_{0}^{\prime}=p_{0}+H_{0} ; \\
& H_{\mu}^{\prime}=p_{\mu}+H_{\mu} .
\end{aligned}
$$

with $q_{0}=t$ and $S$ being the action. The equations of motion are obtained as total differential equations in many variables such as,

$$
\begin{gathered}
\mathrm{d} q_{a}=\frac{\partial H_{\alpha}^{\prime}}{\partial p_{a}} \mathrm{~d} t_{\alpha}, \\
\mathrm{d} p_{\beta}=\frac{\partial H_{\alpha}^{\prime}}{\partial q_{\beta}} \mathrm{d} t_{\alpha}, \\
\mathrm{d} Z=\left(-H_{\alpha}+p_{a} \frac{\partial H_{\alpha}^{\prime}}{\partial p_{a}}\right) \mathrm{d} t_{\alpha} .
\end{gathered}
$$

where $Z=S\left(t_{\alpha}, q_{a}\right)$. These equations are integrable if and only if [34] [35]

$$
\begin{gathered}
\mathrm{d} H_{0}^{\prime}=0, \\
\mathrm{~d} H_{\mu}=0, \mu=n-r+1, \cdots, n .
\end{gathered}
$$

If the conditions (13) and (14) are not satisfied identically, we consider them as new constraints and we examine their variations. Thus repeating this procedure, one may obtain a set of constraints such that all the variations vanish, then we may solve the equations of motion (10) and (11) to get the canonical phasespace coordinates as 


$$
q_{a} \equiv q_{a}\left(t, t_{\mu}\right), \quad p_{a} \equiv p_{a}\left(t, t_{\mu}\right), \quad \mu=1, \cdots, r .
$$

In this case, the path integral representation may be written as

$$
\begin{aligned}
\langle\text { Out }|S| \operatorname{In}\rangle & =\int \prod_{a=1}^{n-r} \mathrm{~d} q^{a} \mathrm{~d} p^{a} \exp \left[i \int_{t_{\alpha}}^{t_{\alpha}^{\prime}}\left(-H_{\alpha}+p_{a} \frac{\partial H_{\alpha}^{\prime}}{\partial p_{a}}\right) \mathrm{d} t_{\alpha}\right], \\
a & =1, \cdots, n-r, \quad \alpha=0, n-r+1, \cdots, n .
\end{aligned}
$$

We should notice that the integral (16) is an integration over the canonical phase space coordinates $\left(q_{a}, p_{a}\right)$.

\section{Hamilton-Jacobi Formulation of Doubly Supersymmetric Models}

The supersymmetric invariant action reproduces to desired a new class of superstring models that possess both spacetime and world-sheet supersymmetries [31] [32] [33].

$$
S=\frac{1}{2} \int \mathrm{d} t\left[e^{-1} \dot{\omega}^{2}-2 e^{-1} \psi \lambda^{m} \dot{\omega}-2 i\left(\dot{\omega}^{m}-\psi \lambda^{m}\right) \bar{\varphi} \gamma_{m} \varphi-e\left(\bar{\varphi} \gamma^{m} \varphi\right)^{2}+\lambda \dot{\lambda}\right] .
$$

where $\mathrm{d} \omega_{m}=\mathrm{d} x_{m}-i\left(\mathrm{~d} \theta \gamma_{m} \bar{\theta}-\theta \gamma_{m} \mathrm{~d} \bar{\theta}\right), m=1,2,3, \cdots, d-1$.

The Lagrangian is

$$
\begin{aligned}
L= & \frac{1}{2 e}\left[\dot{x}_{m}-i\left(\dot{\theta} \gamma_{m} \bar{\theta}-\theta \gamma_{m} \dot{\bar{\theta}}\right)\right]^{2}-\frac{1}{e} \psi \lambda^{m}\left[\dot{x}_{m}-i\left(\dot{\theta} \gamma_{m} \bar{\theta}-\theta \gamma_{m} \dot{\bar{\theta}}\right)\right] \\
& -i\left[\dot{x}^{m}-i\left(\dot{\theta} \gamma^{m} \bar{\theta}-\theta \gamma^{m} \dot{\bar{\theta}}\right)-\psi \lambda^{m}\right] \bar{\varphi} \gamma_{m} \varphi-\frac{e}{2}\left(\bar{\varphi} \gamma^{m} \varphi\right)^{2}+\frac{1}{2} \lambda \dot{\lambda} .
\end{aligned}
$$

The canonical momenta defined in (2) and (3) take the forms

$$
\begin{gathered}
P_{m}=\frac{\partial L}{\partial \dot{x}_{m}}=\frac{1}{e}\left(\dot{x}_{m}-i\left(\dot{\theta} \gamma_{m} \bar{\theta}-\theta \gamma_{m} \dot{\bar{\theta}}\right)\right)-\frac{1}{e} \psi \lambda_{m}-i \bar{\varphi} \gamma_{m} \varphi, \\
\pi_{\theta}=\frac{\partial L}{\partial \dot{\theta}}=-i P_{m} \gamma^{m} \bar{\theta}=-H_{\theta}, \\
\bar{\pi}_{\bar{\theta}}=\frac{\partial L}{\partial \dot{\bar{\theta}}}=i \theta \gamma^{m} P_{m}=-H_{\bar{\theta}}, \\
P_{\psi}=\frac{\partial L}{\partial \dot{\psi}}=0=-H_{\psi}, \\
P_{e}=\frac{\partial L}{\partial \dot{e}}=0=-H_{e}, \\
\pi_{\varphi}=\frac{\partial L}{\partial \dot{\varphi}}=0=-H_{\varphi}, \\
\bar{\pi}_{\bar{\phi}}=\frac{\partial L}{\partial \dot{\bar{\varphi}}}=0=-H_{\bar{\varphi}}, \\
\pi_{\lambda}=\frac{\partial L}{\partial \dot{\lambda}}=\frac{1}{2} \lambda=-H_{\lambda} .
\end{gathered}
$$

We can solve Equation (19) for $\dot{x}_{m}$ in terms $P_{m}$ of other coordinates as 


$$
\dot{x}_{m}=e P_{m}+i\left(\dot{\theta} \gamma_{m} \bar{\theta}-\theta \gamma_{m} \dot{\bar{\theta}}\right)+\psi \lambda_{m}+i e \bar{\varphi} \gamma^{m} \varphi
$$

The canonical Hamiltonian $H_{0}$ takes the form

$$
H_{0}=\frac{1}{2} e p^{2}+P_{m} \psi \lambda^{m}+i e P_{m} \bar{\varphi} \gamma^{m} \varphi+\frac{e}{2}\left(\bar{\varphi} \gamma^{m} \varphi\right)^{2} .
$$

Following the Hamilton-Jacobi formalism, we obtain the set of HJPDE's as

$$
\begin{gathered}
H_{0}^{\prime}=P_{0}+\frac{1}{2} e p^{2}+P_{m} \psi \lambda^{m}+i e P_{m} \bar{\varphi} \gamma^{m} \varphi+\frac{e}{2}\left(\bar{\varphi} \gamma^{m} \varphi\right)^{2}, \\
H_{\theta}^{\prime}=\pi_{\theta}+i P_{m} \gamma^{m} \bar{\theta}, \\
H_{\bar{\theta}}^{\prime}=\bar{\pi}_{\bar{\theta}}-i \theta \gamma^{m} P_{m}, \\
H_{\psi}^{\prime}=P_{\psi}, \\
H_{e}^{\prime}=P_{e}, \\
H_{\varphi}^{\prime}=\pi_{\varphi}, \\
H_{\bar{\varphi}}^{\prime}=\bar{\pi}_{\bar{\varphi}}, \\
H_{\lambda}^{\prime}=\pi_{\lambda}-\frac{1}{2} \lambda .
\end{gathered}
$$

The equations of motion read as

$$
\begin{gathered}
\mathrm{d} x_{m}=\left[e P_{m}+\psi \lambda_{m}+i e \bar{\varphi} \gamma_{m} \varphi\right] \mathrm{d} t+i\left(\mathrm{~d} \theta \gamma_{m} \bar{\theta}-\theta \gamma_{m} \mathrm{~d} \bar{\theta}\right), \\
\mathrm{d} P_{m}=0, \\
\mathrm{~d} \pi_{\theta}=\left(i P_{m} \gamma^{m}\right) \mathrm{d} \bar{\theta}, \\
\mathrm{d} \bar{\pi}_{\bar{\theta}}=\left(-i \gamma^{m} P_{m}\right) \mathrm{d} \theta, \\
\mathrm{d} P_{\psi}=\left(-P_{m} \lambda^{m}\right) \mathrm{d} t, \\
\mathrm{~d} P_{e}=-\left[\frac{1}{2} p^{2}+i P_{m} \bar{\varphi} \gamma^{m} \varphi+\frac{1}{2}\left(\bar{\varphi} \gamma^{m} \varphi\right)^{2}\right] \mathrm{d} t, \\
\mathrm{~d} \pi_{\varphi}=-\left[i e P_{m} \bar{\varphi} \gamma^{m}\right] \mathrm{d} t, \\
\mathrm{~d} \bar{\pi}_{\bar{\varphi}}=-\left[i e P_{m} \gamma^{m} \varphi\right] \mathrm{d} t,
\end{gathered}
$$

and

$$
\mathrm{d} \pi_{\lambda}=-\left(P_{m} \psi\right) \mathrm{d} t
$$

To check the integrability conditions of the system, let us evaluate the total variations of the set of (HJPDE)'s, the variations of

$$
\begin{aligned}
\mathrm{d} H_{0}^{\prime} & =0, \\
\mathrm{~d} H_{\theta}^{\prime} & =0, \\
\mathrm{~d} H_{\bar{\theta}}^{\prime} & =0,
\end{aligned}
$$

are identically zero, whereas

$$
\mathrm{d} H_{\psi}^{\prime}=\left(-P_{m} \lambda^{m}\right) \mathrm{d} t=H_{\psi}^{\prime \prime} \mathrm{d} t
$$




$$
\begin{gathered}
\mathrm{d} H_{e}^{\prime}=-\left[\frac{1}{2} p^{2}+i P_{m} \bar{\varphi} \gamma^{m} \varphi+\frac{1}{2}\left(\bar{\varphi} \gamma^{m} \varphi\right)^{2}\right] \mathrm{d} t=H_{e}^{\prime \prime} \mathrm{d} t, \\
\mathrm{~d} H_{\varphi}^{\prime}=-\left[i e P_{m} \bar{\varphi} \gamma^{m}\right] \mathrm{d} t=H_{\varphi}^{\prime \prime} \mathrm{d} t, \\
\mathrm{~d} H_{\bar{\varphi}}^{\prime}=-\left[i e P_{m} \gamma^{m} \varphi\right] \mathrm{d} t=H_{\bar{\varphi}}^{\prime \prime} \mathrm{d} t, \\
\mathrm{~d} H_{\lambda}^{\prime}=-\left(P_{m} \psi\right) \mathrm{d} t=H_{\lambda}^{\prime \prime} \mathrm{d} t .
\end{gathered}
$$

are not identically zero, and hence we obtain the following new constraints

$$
\begin{gathered}
H_{\psi}^{\prime \prime}=-P_{m} \lambda^{m}, \\
H_{e}^{\prime \prime}=-\left[\frac{1}{2} p^{2}+i P_{m} \bar{\varphi}^{m} \varphi+\frac{1}{2}\left(\bar{\varphi} \gamma^{m} \varphi\right)^{2}\right], \\
H_{\varphi}^{\prime \prime}=-\left[i e P_{m} \bar{\varphi} \gamma^{m}\right], \\
H_{\bar{\varphi}}^{\prime \prime}=-\left[i e P_{m} \gamma^{m} \varphi\right], \\
H_{\lambda}^{\prime \prime}=-\left(P_{m} \psi\right) .
\end{gathered}
$$

We notice that the total differential of new set of constraints (54)-(58) vanish identically, i.e.

$$
\begin{aligned}
\mathrm{d} H_{\psi}^{\prime \prime} & =0, \\
\mathrm{~d} H_{e}^{\prime \prime} & =0, \\
\mathrm{~d} H_{\varphi}^{\prime \prime} & =0, \\
\mathrm{~d} H_{\bar{\varphi}}^{\prime \prime} & =0, \\
\mathrm{~d} H_{\lambda}^{\prime \prime} & =0 .
\end{aligned}
$$

Thus, the equations of motion (37)-(45) and the new constraints (54)-(58) represent an integrable system. According to (12) the action can be written as

$$
S=\frac{1}{2} \int\left[e P^{2}-e\left(\bar{\varphi} \gamma^{m} \varphi\right)^{2}+\lambda \dot{\lambda}\right] \mathrm{d} t .
$$

in this case, the path integral of the system takes the form

$$
\left\langle x^{m}, t ; x^{\prime m}, t^{\prime}\right\rangle=\int \mathrm{d} x^{m} \mathrm{~d} P^{m} \exp \left\{i \frac{1}{2} \int\left[e P^{2}-e\left(\bar{\varphi} \gamma^{m} \varphi\right)^{2}+\lambda \dot{\lambda}\right] \mathrm{d} t\right\} .
$$

\section{Conclusion}

In this paper, we have addressed the path integral quantization for constrained systems, to the derivation of quantum physics from classical physics. We have examined the double supersymmetric models with the spinning superparticle in the component and superfield form by applying the Hamilton-Jacobi formulation. One notices that our formalism does not depend on the N-extended supersymmetric which introduces extra degrees of freedom. In Hamilton-Jacobi formalism of the classical constraints, no need for gauge fixing of first-class constraints, no need to eliminate second-class constraints, such as in Dirac method, consequently there is no difficulty in treating the constraints. As a strategy, we 
first work out the constraints as a set of Hamilton-Jacobi partial differential equations (HJPDE), then we obtained the equations of motion as total differential equations in many variables, which require the investigation of integrability conditions. The canonical path integral quantization has been done, since the system is integrable, the integration is taken over the canonical phase space.

\section{Conflicts of Interest}

The authors declare no conflicts of interest regarding the publication of this paper.

\section{References}

[1] Brink, L. and Schwarz, J.H. (1981) Quantum Superspace. Physics Letter B, 100, 310-312. https://doi.org/10.1016/0370-2693(81)90093-9

[2] Galajinsky, A.V. and Gitman, D.M. (1998) Siegel Superparticle, Higher-Order Fermionic Constraints, and Path Integrals. Nuclear Physics B, 536, 435-453.

https://doi.org/10.1016/S0550-3213(98)00584-7

[3] Grassi, P.A., Policastro, G. and van Nieuwenhuizen, P. (2003) The Covariant Quantum Superstring and Superparticle from Their Classical Actions. Physics Letters $B$, 553, 96-104. https://doi.org/10.1016/S0370-2693(02)03185-4

[4] Deriglazov, A.A., Galajinsky, A.V. and Lyakhovich, S.L. (1996) Weak Dirac bracket Construction and the Superparticle Covariant Quantization Problem. Nuclear Physics $B$, 473, 245-266. https://doi.org/10.1016/0550-3213(96)00229-5

[5] Brink, L., Henneaux, M. and Teitelboim, C. (1987) Covariant Hamiltonian Formulation of the Superparticle. Nuclear Physics B, 293, 505-540.

https://doi.org/10.1016/0550-3213(87)90082-4

[6] Delduc, F., Galperin, A. and Sokatchev, E. (1992) Lorentz-Harmonic (Super)Fields and (Super)Particles. Nuclear Physics B, 368, 143-171. https://doi.org/10.1016/0550-3213(92)90201-L

[7] Dirac, P.A.M. (1950) Gereralized Hamiltonian Dynamics. Canadian Journal of Mathematics, 2, 129-148. https://doi.org/10.4153/CJM-1950-012-1

[8] Dirac P.A.M. (1964) Lectures on Quantum Mechanics. Belfer Graduate School of Science, Yeshiva University, New York.

[9] Carathéodory, C. (1967) Calculus of Variations and Partial Differential Equations of First Order, Part II: Calculus of Variations. Holden-Day Inc., San Francisco.

[10] Güler, Y. (1992) Canonical Formulation of Singular Systems. Il Nuovo Cimento B, 107, 1389-1395. https://doi.org/10.1007/BF02722849

[11] Güler, Y. (1992) Integration of Singular Systems. Il Nuovo Cimento B, 107, 1143-1149. https://doi.org/10.1007/BF02727199

[12] Faddeev, L.D. and Popov, V.M. (1967) Feynman Diagrams for the Yang-Mills Field. Physics Letters B, 25, 29-30. https://doi.org/10.1016/0370-2693(67)90067-6

[13] Senjanovic, P. (1976) Path Integral Quantization of Field Theories with Second-Class Constraints. Annals of Physics, 100, 221-261. https://doi.org/10.1016/0003-4916(76)90062-2

[14] Fradkin, E.S. and Vilkovisky, G.A. (1973) S Matrix for Gravitational Field. II. Local Measure; General Relations; Elements of Renormalization Theory. Physical Review D, 8, 4241-4285. https://doi.org/10.1103/PhysRevD.8.4241 
[15] Fradkin, E.S. and Vilkovisky, G.A. (1975) Quantization of Relativistic Systems with Constraints. Physics Letters B, 55, 224-226. https://doi.org/10.1016/0370-2693(75)90448-7

[16] Muslih, S.I. (2000) Path Integral Quantization of Electromagnetic Theory. Il Nuovo Cimento $B, 115,7-12$.

[17] Farahat, N.I. and Güler, Y. (1996) Treatment of a Relativistic Particle in External Electromagnetic Field as a Singular System. Il Nuovo Cimento B, 111, 513-520. https://doi.org/10.1007/BF02724560

[18] Rabei, E.M. and Tawfiq, S. (1997) Hamilton-Jacobi Treatment of QED and Yang-Mills Theory as Constrained Systems. Hadronic Journal, 20, 232-249.

[19] Muslih, S.I. (2002) Canonical Path Integral Quantization of Einstein's Gravitational Field. General Relativity and Gravitation, 34, 1059-1065. https://doi.org/10.1023/A:1016561904569

[20] Farahat, N.I. and Nassar, Z. (2005) Relativistic Classical Spinning Particle as Singular System of Second Order. Islamic University Journal, 13, 239-248.

[21] Farahat, N.I. and Nassar, Z. (2002) Treatment of a Spinning Particle or Super-Gravity in One Dimension Singular System. Hadronic Journal, 25, 239-249.

[22] Muslih, S.I. and Güler, Y. (1998) Is Gauge Fixing of Constrained Systems Necessary? Nuovo Cimento B, 113, 277-289.

[23] Muslih, S.I. and Güler, Y. (1997) The Feynman Path Integral Quantization of Constrained Systems. Il Nuovo Cimento B, 112, 97-107.

[24] Muslih, S.I. (2002) Rdeduced Phase-Space Quantization of Constrained Systems. II Nuovo Cimento B, 117, 383-391.

[25] Farahat, N.I. and Elegla, H.A. (2006) Hamilton-Jacobi Formulation of Siegle Superparticle. Turkish Journal of Physics, 30, 473-478.

[26] Elegla, H.A. and Farahat, N.I. (2008) Path Integral Quantization of Spinning Superparticle. Turkish Journal of Physics, 32, 261-267.

[27] Farahat, N.I. and Elegla, H.A. (2008) Path Integral Quantization of Brink-Schwarz Superparticle. Electronic Journal of Theoretical Physics, 19, 57-64.

[28] Farahat, N.I. and Elegla, H.A. (2010) Path Integral Quantization of Superstring. Modern Physics Letters A, 25, 135-141. https://doi.org/10.1142/S0217732310032287

[29] Farahat, N.I. and Elegla, H.A. (2010) Hamilton-Jacobi Formulation of Green-Schwarz. International Journal of Theoretical Physics, 49, 384-394. https://doi.org/10.1007/s10773-009-0214-8

[30] Farahat, N.I. and Elegla, H.A. (2013) Path Integral Quantization of Superparticle with 1/4 Supersymmetry Breaking. Journal of Applied Mathematics and Physics, 1, 105-109. https://doi.org/10.4236/jamp.2013.15016

[31] Gates Jr., S.J. and Nishino, H. (1986) D=2 Superfield Supergravity, Local (Supersymmetry) ${ }^{2}$ and Non-Linear $\Sigma$ Models. Classical and Quantum Gravity, 3, 391-399. https://doi.org/10.1088/0264-9381/3/3/013

[32] Galajinsky, A. and Lechtenfeld, O. (1999) Towards a Stringy Extension of Self-Dual Super Yang-Mills. Physics Letters B, 460, 288-294. https://doi.org/10.1016/S0370-2693(99)00754-6

[33] Frydryszak, A.M. (1996) Lagrangian Models of the Particles with Spin: The First Seventy Years. In: Jancewicz, B., Ed., From Field Theory to Quantum Groups, World Scientific, Singapore, 151-172. https://doi.org/10.1142/9789812830425_0009

[34] Muslih, S.I. (2003) Completely and Partially Integrable Systems of Total Differential 
Equations. Il Nuovo Cimento B, 118, 505.

[35] Muslih, S.I. (2004) Integrability and Action Function in Multi-Hamiltonian Systems. Modern Physics Letter A, 19, 863-870.

https://doi.org/10.1142/S0217732304013751 